\title{
ON EVALUATION FORMULAS FOR DOUBLE L-VALUES
}

\author{
HIROFUMI TSUMURA
}

\begin{abstract}
In this paper, we give some evaluation formulas for the values of double $L$-series of Tornheim's type, in terms of the Dirichlet $L$-values and the Riemann zeta values at positive integers. As special cases, these give the formulas for double $L$-values given by Terhune.
\end{abstract}

\section{INTRODUCTION}

Let $\mathbb{N}$ be the set of natural numbers, $\mathbb{N}_{0}=\mathbb{N} \cup\{0\}, \mathbb{Z}$ the ring of rational integers, $\mathbb{Q}$ the field of rational numbers, $\mathbb{R}$ the field of real numbers and $\mathbb{C}$ the field of complex numbers.

Let $\chi, \psi$ be primitive Dirichlet characters. We consider the duuble $L$-series of Tornheim's type defined by

$$
\mathcal{L}(k, l, d ; \chi, \psi)=\sum_{m, n=1}^{\infty} \frac{\chi(m) \psi(m+n)}{m^{k} n^{l}(m+n)^{d}},
$$

where $k, l, d \in \mathbb{N}_{0}$ with $k+d>1, l+d>1, k+l+d>2$. This can be a character analogue of the Tornheim double series

$$
T(k, l, d)=\sum_{m, n=1}^{\infty} \frac{1}{m^{k} n^{l}(m+n)^{d}}
$$

defined in [8].

Tornheim showed that $T(k, l, N-k-l)$ can be expressed as a polynomial in $\{\zeta(j) \mid 2 \leqslant j \leqslant N\}$ with rational coefficients when $N$ is odd and $N \geqslant 3$, where $\zeta(s)$ is the Riemann zeta function. This essentially includes Euler's consideration for $T(k, 0, N$ - $k$ ) which is called the Euler sum (see, for example, [2]). Independently, Mordell also considered these series in [5]. Recently Huard, Williams and Zhang Nan-Yue gave an explicit formula for $T(k, l, N-k-l)$ as a rational linear combination of the products $\zeta(2 j) \zeta(N-2 j)(0 \leqslant j \leqslant(N-3) / 2)$ when $N$ is odd, $N \geqslant 3$, and $k, l \in \mathbb{N}_{0}$ satisfying $1 \leqslant k+l \leqslant N-1, k \leqslant N-2$ and $l \leqslant N-2$ (see [3]). Recently Matsumoto considered

Received 17th February, 2004

Copyright Clearance Centre, Inc. Serial-fee code: 0004-9727/04 \$A2.00+0.00. 
$T\left(s_{1}, s_{2}, s_{3}\right)$ as a meromorphic function of complex variables $\left(s_{1}, s_{2}, s_{3}\right) \in \mathbb{C}^{3}$ in [4]. Note that he further considered multiple zeta functions of Tornheim's type.

$\mathcal{L}(k, 0, d ; \chi, \psi)$ is what is called the double $L$-series (see, for example, $[1,6,7])$. Recently Terhune proved that if $\chi(-1) \psi(-1)=(-1)^{k+d+1}$ then $\mathcal{L}(k, 0, d ; \chi, \psi)$ is a polynomial in the values of polylogarithms at $p$ th roots of unity with $\mathbb{Q}\left(\zeta_{m}\right)$-coefficients, where $m, p \in \mathbb{N}$ are determined by $\chi, \psi$ (see $[6,7]$ ). As concrete examples, he listed some evaluation formulas for $\mathcal{L}\left(k, 0, d ; \chi_{3}, \chi_{0}\right), \mathcal{L}\left(k, 0, d ; \chi_{0}, \chi_{3}\right)$ and $\mathcal{L}\left(k, 0, d ; \chi_{5}, \chi_{0}\right)$ when $k+d$ is even and odd, respectively, where $\chi_{0}$ is the trivial character, $\chi_{3}$ and $\chi_{5}$ are the quadratic character of conductor 3 and 5 , respectively.

The aim of this paper is to give explicit evaluation formulas for $\mathcal{L}\left(k, l, d ; \chi_{0}, \chi\right)$ for an arbitrary primitive Dirichlet character $\chi \neq \chi_{0}$, when $\chi(-1)=(-1)^{k+l+d+1}$ (see Theorem 3.1). In particular when $l=0$, we obtain evaluation formulas for double $L$-series $\mathcal{L}\left(k, 0, d ; \chi_{0}, \chi\right)$ and $\mathcal{L}\left(k, 0, d ; \chi, \chi_{0}\right)$, when $\chi(-1)=(-1)^{k+d+1}$. These include Terhune's formulas for double $L$-series given in $[6,7]$.

In order to prove our assertion, we make use of our previous result, namely the evaluation formulas for

$$
\sum_{m, n=1}^{\infty} \frac{(-1)^{m+n} \sin ((m+n) \theta)}{m^{k} n^{l}(m+n)^{d}}
$$

for $\theta \in[-\pi, \pi]$ when $k+l+d$ is even (see [9, Proposition 3.1]).

As concrete examples, we give evaluation formulas for $\mathcal{L}\left(k, l, d ; \chi_{0}, \chi_{3}\right)$ when $k+l+d$ is even, in terms of the values of $\zeta(s)$ and the Dirichlet $L$-series $L\left(s, \chi_{3}\right)$ at positive integers (see Proposition 4.1). For example, we have

$$
\begin{aligned}
& \mathcal{L}\left(1,1,2 ; \chi_{0}, \chi_{3}\right)=2 L\left(4, \chi_{3}\right)-\frac{26 \sqrt{3}}{81} \pi \zeta(3), \\
& \mathcal{L}\left(2,0,2 ; \chi_{3}, \chi_{0}\right)=L\left(4, \chi_{3}\right)+\frac{2 \pi^{2}}{9} L\left(2, \chi_{3}\right)-\frac{26 \sqrt{3}}{81} \pi \zeta(3) .
\end{aligned}
$$

Note that $L\left(1, \chi_{3}\right)=\sqrt{3} \pi / 9$. (1.4) was obtained by Terhune.

\section{Preliminaries}

In this section, we quote some results from [9] as follows. For $u \in \mathbb{R}$ with $1 \leqslant u$ $\leqslant 1+\delta$ and $s \in \mathbb{R}$, we define

$$
\phi(s ; u):=\sum_{m=1}^{\infty} \frac{(-u)^{-m}}{m^{s}}
$$

If $u>1$ then $\phi(s ; u)$ is convergent absolutely for any $s \in \mathbb{R}$. In the case when $u=1$, let $\phi(s):=\phi(s ; 1)=\left(2^{1-s}-1\right) \zeta(s)$. 
We denote the $p$ th derivative of $\sin (X)$ by $\sin ^{(p)}(X)$. Furthermore we denote $\left.\sin ^{(p)}(X)\right|_{X=m \theta}$ by $\sin ^{(p)}(m \theta)$ for $m \in \mathbb{N}_{0}$. Then we define

$$
\mathcal{I}_{p}(\theta ; k ; u):=\sum_{m=1}^{\infty} \frac{(-u)^{-m} \sin ^{(p)}(m \theta)}{m^{k}}
$$

for $p \in \mathbb{N}_{0}, k \in \mathbb{N}, \theta \in[-\pi, \pi]$ and $u \in[1,1+\delta]$. Let $\lambda_{j}=\left\{1+(-1)^{j}\right\} / 2$ for $j \in \mathbb{Z}$. Then we have the following lemma (see [9, Proposition 3.1]).

LEMMA 2.1. Let $k, l, d \in \mathbb{N}_{0}$ with $k+d>1, l+d>1, k+l+d>2, d \geqslant 2$ and $\theta \in[-\pi, \pi]$. Suppose $k+l+d \equiv 0(\bmod 2)$. Then

$$
\begin{aligned}
\sum_{m, n=1}^{\infty} & \frac{(-1)^{m+n} \sin ((m+n) \theta)}{m^{k} n^{l}(m+n)^{d}} \\
= & \sum_{j=0}^{k} \phi(k-j)(-1)^{j} \lambda_{k+j} \sum_{\nu=0}^{j}\left(\begin{array}{c}
d-1+j-\nu \\
j-\nu
\end{array}\right) \frac{(-\theta)^{\nu}}{\nu !} \mathcal{I}_{\nu}(\theta ; d+j+l-\nu ; 1) \\
& +\sum_{j=0}^{l} \phi(l-j)(-1)^{j} \lambda_{l+j} \sum_{\nu=0}^{j}\left(\begin{array}{c}
d-1+j-\nu \\
j-\nu
\end{array}\right) \frac{(-\theta)^{\nu}}{\nu !} \mathcal{I}_{\nu}(\theta ; d+j+k-\nu ; 1) \\
& +\sum_{n=0}^{[(d-2) / 2]} \beta_{2 n+1-d}(k, l ; 1) \frac{(-1)^{n} \theta^{2 n+1}}{(2 n+1) !}
\end{aligned}
$$

where

$$
\begin{aligned}
\beta_{-N-1}(k, l ; 1) \lambda_{k+l+N}= & -2 \sum_{\nu=0}^{N} \phi(N-\nu) \lambda_{N+\nu} \lambda_{k+l+\nu} \\
& \times\left\{(-1)^{k} \sum_{\rho=0}^{[k / 2]} \phi(2 \rho) \sum_{\mu=0}^{[(k-2 \rho-1) / 2]}\left(\begin{array}{l}
\nu+k-2 \rho-2 \mu \\
k-2 \rho-2 \mu-1
\end{array}\right)\right. \\
& \times \zeta(k+l+\nu-2 \rho-2 \mu+1) \frac{(-1)^{\mu} \pi^{2 \mu}}{(2 \mu+1) !} \\
& +(-1)^{l} \sum_{\rho=0}^{[l / 2]} \phi(2 \rho) \sum_{\mu=0}^{[(l-2 \rho-1) / 2]}\left(\begin{array}{l}
\nu+l-2 \rho-2 \mu \\
l-2 \rho-2 \mu-1
\end{array}\right) \\
& \left.\times \zeta(k+l+\nu-2 \rho-2 \mu+1) \frac{(-1)^{\mu} \pi^{2 \mu}}{(2 \mu+1) !}\right\}
\end{aligned}
$$

for $N \in \mathbb{N}_{0}$.

Proof: It follows from Lemma 2.2 in [9] that each side of Equation (3.1) in [9] is uniformly convergent with respect to $u \in[1,1+\delta]$ because $d \geqslant 2$ and $\theta \in[-\pi, \pi]$. So (3.1) in [9] holds for $u=1$. Note that we assumed $k, l, d \in \mathbb{N}$ in Proposition 3.1 of [9]. However, we can see that $[9$, Proposition 3.1$]$ holds for $k, l, d \in \mathbb{N}_{0}$ satisfying the 
conditions in the statement of Lemma 2.1. Hence we obtain (2.2). From (3.6) in [9], we obtain (2.3).

When $d \geqslant 3$, we can differentiate (2.2) with respect to $\theta$ because of its uniform convergency. Using the known relation

$$
-\left(\begin{array}{c}
x-1 \\
y-1
\end{array}\right)+\left(\begin{array}{l}
x \\
y
\end{array}\right)=\left(\begin{array}{c}
x-1 \\
y
\end{array}\right)
$$

and replacing $d-1$ with $d$, we have the following.

LEMMA 2.2. Let $k, l, d \in \mathbb{N}_{0}$ with $k+d>1, l+d>1, k+l+d>2, d \geqslant 2$ and $\theta \in[-\pi, \pi]$. Suppose $k+l+d \equiv 1(\bmod 2)$. Then

$$
\begin{aligned}
\sum_{m, n=1}^{\infty} & \frac{(-1)^{m+n} \cos ((m+n) \theta)}{m^{k} n^{l}(m+n)^{d}} \\
= & \sum_{j=0}^{k} \phi(k-j)(-1)^{j} \lambda_{k+j} \sum_{\nu=0}^{j}\left(\begin{array}{c}
d-1+j-\nu \\
j-\nu
\end{array}\right) \frac{(-\theta)^{\nu}}{\nu !} \mathcal{I}_{\nu+1}(\theta ; d+j+l-\nu ; 1) \\
& +\sum_{j=0}^{l} \phi(l-j)(-1)^{j} \lambda_{l+j} \sum_{\nu=0}^{j}\left(\begin{array}{c}
d-1+j-\nu \\
j-\nu
\end{array}\right) \frac{(-\theta)^{\nu}}{\nu !} \mathcal{I}_{\nu+1}(\theta ; d+j+k-\nu ; 1) \\
& +\sum_{n=0}^{[(d-1) / 2\rceil} \beta_{2 n-d}(k, l ; 1) \frac{(-1)^{n} \theta^{2 n}}{2 n !} .
\end{aligned}
$$

For simplicity, for $p \in\{0,1\}$, we let

$$
\begin{aligned}
\mathcal{A}_{p}(\theta ; k, l, d)= & \sum_{j=0}^{k} \phi(k-j)(-1)^{j} \lambda_{k+j} \\
& \times \sum_{\nu=0}^{j}\left(\begin{array}{c}
d-1+j-\nu \\
j-\nu
\end{array}\right) \frac{(-\theta)^{\nu}}{\nu !} \mathcal{I}_{\nu+p}(\theta ; d+j+l-\nu ; 1) \\
& \quad+\sum_{j=0}^{l} \phi(l-j)(-1)^{j} \lambda_{l+j} \\
& \times \sum_{\nu=0}^{j}\left(\begin{array}{c}
d-1+j-\nu \\
j-\nu
\end{array}\right) \frac{(-\theta)^{\nu}}{\nu !} \mathcal{I}_{\nu+p}(\theta ; d+j+k-\nu ; 1) .
\end{aligned}
$$

ExAMPLE 2.3. Putting $(k, l)=(1,1)$ in $(2.3)$, we have $\beta_{-1}(1,1 ; 1)=\zeta(3)$. Furthermore, putting $(k, l)=(2,0)$, we have $\beta_{-1}(2,0 ; 1)=-\zeta(3)$.

\section{EVAluation formulas}

Let $\chi$ be the primitive Dirichlet character with conductor $f>1$. It is well-known that

$$
\chi(n)=\frac{1}{\tau(\bar{\chi})} \sum_{a=1}^{f} \bar{\chi}(a) e^{2 \pi i a n / f}
$$


for $n \in \mathbb{Z}$, where $\bar{\chi}=\chi^{-1}$ and $\tau(\chi)=\sum_{a=1}^{f} \chi(a) e^{2 \pi i a / f}$ (see, for example, [10, Lemma 4.7]). Hence we have

$$
\begin{array}{ll}
\chi(n)=\frac{2}{\tau(\bar{\chi})} \sum_{a=1}^{[J / 2]} \bar{\chi}(a) \cos (2 \pi a n / f) & (\text { if } \chi(-1)=1), \\
\chi(n)=\frac{2 i}{\tau(\bar{\chi})} \sum_{a=1}^{[f / 2]} \bar{\chi}(a) \sin (2 \pi a n / f) & (\text { if } \chi(-1)=-1) .
\end{array}
$$

Furthermore we can check that if $f$ is even then

$$
\begin{aligned}
& \sin \left(\frac{2 \pi n}{f}\left(\frac{f}{2}-b\right)\right)=(-1)^{n} \sin \left(\frac{2 \pi n b}{f}\right), \\
& \cos \left(\frac{2 \pi n}{f}\left(\frac{f}{2}-b\right)\right)=(-1)^{n} \cos \left(\frac{2 \pi n b}{f}\right),
\end{aligned}
$$

and if $f$ is odd then

$$
\begin{aligned}
& \sin \left(\frac{2 \pi n}{f}\left(\frac{f+1}{2}-b\right)\right)=(-1)^{n} \sin \left(\frac{2 \pi n}{f}\left(b-\frac{1}{2}\right)\right), \\
& \cos \left(\frac{2 \pi n}{f}\left(\frac{f+1}{2}-b\right)\right)=(-1)^{n} \cos \left(\frac{2 \pi n}{f}\left(b-\frac{1}{2}\right)\right) .
\end{aligned}
$$

For $k \in \mathbb{N}$ and $z \in \mathbb{C}$ with $|z| \leqslant 1$, we consider the polylogarithms defined by

$$
L i(k ; z)=\sum_{m=1}^{\infty} \frac{z^{m}}{m^{k}} .
$$

From the relation

$$
\sin ^{(p)} x=\frac{i^{p-1}}{2}\left(e^{i x}+(-1)^{p-1} e^{-i x}\right)
$$

we have

$$
\mathcal{I}_{p}(\theta ; k ; 1)=\frac{i^{p-1}}{2}\left\{L i\left(k ;-e^{i \theta}\right)+(-1)^{p-1} L i\left(k ;-e^{-i \theta}\right)\right\}
$$

for $p \in \mathbb{N}_{0}$.

TheOREM 3.1. Let $k, l, d \in \mathbb{N}_{0}$ with $k+d>1, l+d>1, k+l+d>2$ and $d \geqslant 2$, and $\chi$ be a Dirichlet character with conductor $f>1$ and with $\chi(-1)=(-1)^{k+l+d+1}$. If $\chi(-1)=1$ and $f$ is even, then

$$
\begin{aligned}
\mathcal{L}\left(k, l, d ; \chi_{0}, \chi\right)=\frac{2}{\tau(\bar{\chi})} \sum_{b=1}^{f / 2-1} \bar{\chi}\left(\frac{f}{2}-b\right) & \left\{\mathcal{A}_{1}\left(\frac{2 \pi b}{f} ; k, l, d\right)\right. \\
& \left.+\sum_{n=0}^{[(d-1) / 2]} \beta_{2 n-d}(k, l ; 1) \frac{(-1)^{n}(2 \pi b / f)^{2 n}}{2 n !}\right\}
\end{aligned}
$$


If $\chi(-1)=-1$ and $f$ is even, then

$$
\begin{aligned}
\mathcal{L}\left(k, l, d ; \chi_{0}, \chi\right)=\frac{2 i}{\tau(\bar{\chi})} & \sum_{b=1}^{J / 2-1} \bar{\chi}\left(\frac{f}{2}-b\right)\left\{\mathcal{A}_{0}\left(\frac{2 \pi(b-1 / 2)}{f} ; k, l, d\right)\right. \\
& \left.+\sum_{n=0}^{[(d-2) / 2]} \beta_{2 n+1-d}(k, l ; 1) \frac{(-1)^{n}(2 \pi(b-1 / 2) / f)^{2 n+1}}{(2 n+1) !}\right\} .
\end{aligned}
$$

If $\chi(-1)=1$ and $f$ is odd, then

$(3.11) \mathcal{L}\left(k, l, d ; \chi_{0}, \chi\right)=\frac{2}{\tau(\bar{\chi})} \sum_{b=1}^{(f-1) / 2} \bar{\chi}\left(\frac{f+1}{2}-b\right)$

$$
\times\left\{\mathcal{A}_{1}\left(\frac{2 \pi b}{f} ; k, l, d\right)+\sum_{n=0}^{[(d-1) / 2]} \beta_{2 n-d}(k, l ; 1) \frac{(-1)^{n}(2 \pi b / f)^{2 n}}{2 n !}\right\} .
$$

If $\chi(-1)=-1$ and $f$ is odd, then

$$
\begin{aligned}
\mathcal{L}\left(k, l, d ; \chi_{0}, \chi\right)= & \frac{2 i}{\tau(\bar{\chi})} \sum_{b=1}^{(f-1) / 2} \bar{\chi}\left(\frac{f+1}{2}-b\right) \\
& \times\left\{\mathcal{A}_{0}\left(\frac{2 \pi(b-1 / 2)}{f} ; k, l, d\right)\right. \\
& \left.\quad+\sum_{n=0}^{[(d-2) / 2]} \beta_{2 n+1-d}(k, l ; 1) \frac{(-1)^{n}(2 \pi(b-1 / 2) / f)^{2 n+1}}{(2 n+1) !}\right\}
\end{aligned}
$$

Note that

(3.13) $\mathcal{A}_{p}(\theta ; k, l, d)$

$$
\begin{aligned}
& =\sum_{j=0}^{k} \phi(k-j)(-1)^{j} \lambda_{k+j} \sum_{\nu=0}^{j}\left(\begin{array}{c}
d-1+j-\nu \\
j-\nu
\end{array}\right) \frac{(-\theta)^{\nu}}{\nu !} \\
& \times \frac{i^{\nu+p-1}}{2}\left\{L i\left(d+j+l-\nu ;-e^{i \theta}\right)+(-1)^{\nu+p-1} L i\left(d+j+l-\nu ;-e^{-i \theta}\right)\right\} \\
& \quad+\sum_{j=0}^{l} \phi(l-j)(-1)^{j} \lambda_{l+j} \sum_{\nu=0}^{j}\left(\begin{array}{c}
d-1+j-\nu \\
j-\nu
\end{array}\right) \frac{(-\theta)^{\nu}}{\nu !} \\
& \times \frac{i^{\nu+p-1}}{2}\left\{L i\left(d+j+k-\nu ;-e^{i \theta}\right)+(-1)^{\nu+p-1} L i\left(d+j+k-\nu ;-e^{-i \theta}\right)\right\},
\end{aligned}
$$

and $\beta_{-j}(k, l ; 1)$ is defined by $(2.3)$.

Proof: If $f$ is even (respecitvely, odd) then we put $a=f / 2-b$ (respectively, $a=(f+1) / 2-b)$ in (3.2) and (3.3). By combining Lemma 2.1, Lemma 2.2 and (3.2)(3.9), we obtain (3.10)-(3.12). 
REMARK 3.2. In particular when $l=0$, we obtain the evaluation formulas for $\mathcal{L}\left(k, 0, d ; \chi_{0}, \chi\right)$ when $\chi(-1)=(-1)^{k+d+1}$. Furthermore, by using

$$
\begin{aligned}
L(p, \chi) \zeta(q) & =\left(\sum_{0<m_{1}<m_{2}}+\sum_{m_{1}>m_{2}>0}+\sum_{0<m_{1}=m_{2}}\right) \frac{\chi\left(m_{1}\right)}{m_{1}^{p} m_{2} q} \\
& =\mathcal{L}\left(p, 0, q ; \chi, \chi_{0}\right)+\mathcal{L}\left(q, 0, p ; \chi_{0}, \chi\right)+L(p+q, \chi),
\end{aligned}
$$

we obtain the evaluation formulas for $\mathcal{L}\left(k, 0, d ; \chi, \chi_{0}\right)$.

\section{THE CASE OF CONDUCTOR 3}

As concrete examples, we give the evaluation formulas for $\mathcal{L}\left(k, l, d ; \chi_{3}\right)$ in terms of the Dirichlet $L$-values and the Riemann zeta values as follows.

Proposition 4.1. Let $k, l, d \in \mathbb{N}_{0}$ with $k+d>1, l+d>1, k+l+d>2$ and $d \geqslant 2$. Suppose $k+l+d$ is even. Then

(4.1) $\mathcal{L}\left(k, l, d ; \chi_{0}, \chi_{3}\right)$

$$
\begin{aligned}
&= \frac{1}{\sqrt{3}}\left[\sum_{j=0}^{k} \phi(k-j)(-1)^{j} \lambda_{k+j}\right. \\
& \times \times \sqrt{3} \sum_{\mu=0}^{[j / 2]}\left(\begin{array}{c}
d-1+j-2 \mu \\
j-2 \mu
\end{array}\right) \frac{(-1)^{\mu}(\pi / 3)^{2 \mu}}{(2 \mu) !} L\left(d+j+l-2 \mu, \chi_{3}\right) \\
&\left.\quad-\sum_{\mu=0}^{[(j-1) / 2]}\left(\begin{array}{c}
d-2+j-2 \mu \\
j-2 \mu-1
\end{array}\right) \frac{(-1)^{\mu}(\pi / 3)^{2 \mu+1}}{(2 \mu+1) !} \psi(d+j+l-2 \mu-1)\right\} \\
&+\sum_{j=0}^{l} \phi(l-j)(-1)^{j} \lambda_{l+j} \\
& \times\left\{\sqrt{3} \sum_{\mu=0}^{[j / 2]}\left(\begin{array}{c}
d-1+j-2 \mu \\
j-2 \mu
\end{array}\right) \frac{(-1)^{\mu}(\pi / 3)^{2 \mu}}{(2 \mu) !} L\left(d+j+k-2 \mu, \chi_{3}\right)\right. \\
&\left.\left.\quad-\sum_{\mu=0}^{[(j-1) / 2]}\left(\begin{array}{c}
d-2+j-2 \mu \\
j-2 \mu-1
\end{array}\right) \frac{(-1)^{\mu}(\pi / 3)^{2 \mu+1}}{(2 \mu+1) !} \psi(d+j+k-2 \mu-1)\right\}\right] \\
& \quad-\frac{2}{\sqrt{3}} \sum_{n=0}^{[(d-2) / 2]} \beta_{2 n+1-d}(k, l ; 1) \frac{(-1)^{n}(\pi / 3)^{2 n+1}}{(2 n+1) !},
\end{aligned}
$$

where $\phi(s)=\left(2^{1-s}-1\right) \zeta(s), \psi(s)=\left(1-3^{1-s}\right) \zeta(s)$.

Proof: From (3.8) and (3.9), we can easily check that

$$
\begin{aligned}
\mathcal{I}_{2 \mu}\left(\frac{\pi}{3} ; k ; 1\right) & =-\frac{\sqrt{3}}{2}(-1)^{\mu} L\left(k, \chi_{3}\right), \\
\mathcal{I}_{2 \mu+1}\left(\frac{\pi}{3} ; k ; 1\right) & =-\frac{1}{2}(-1)^{\mu} \psi(k)
\end{aligned}
$$


for $\mu \in \mathbb{N}_{0}$, where $\psi(s)=\left(1-3^{1-s}\right) \zeta(s)$. By applying Theorem 3.1 with $\chi=\chi_{3}$ and $f=3$, we obtain the assertion.

EXAMPle 4.2. Putting $(k, l)=(1,1)$ in $(2.3)$, we have

$$
\beta_{-2 j-1}(1,1 ; 1)=-2 \sum_{\mu=0}^{j}\left(2^{1-2 j+2 \mu}-1\right) \zeta(2 j-2 \mu) \zeta(2 \mu+3)
$$

for $j \in \mathbb{N}_{0}$, because $\phi(0)=-1 / 2$. Putting $(k, l, d)=(1,1,2 r)$ in $(4.1)$, we have

$$
\begin{aligned}
\mathcal{L}\left(1,1,2 r ; \chi_{0}, \chi_{3}\right)= & \frac{1}{\sqrt{3}}\left\{(2 \sqrt{3} r) L\left(2 r+2, \chi_{3}\right)-\frac{\pi}{3}\left(1-3^{-2 r}\right) \zeta(2 r+1)\right\} \\
& \quad+\frac{4}{\sqrt{3}} \sum_{n=0}^{r-1} \sum_{\mu=0}^{r-1-n}\left(2^{3+2 n+2 \mu-2 r}-1\right) \zeta(2 r-2-2 n-2 \mu) \zeta(2 \mu+3) \\
\times & \frac{(-1)^{n}(\pi / 3)^{2 n+1}}{(2 n+1) !}
\end{aligned}
$$

for $r \in \mathbb{N}$. Putting $r=1$, we obtain (1.3). Furthermore, putting $r=2$, we have

$$
\mathcal{L}\left(1,1,4 ; \chi_{0}, \chi_{3}\right)=4 L\left(6, \chi_{3}\right)-\frac{242 \sqrt{3}}{729} \pi \zeta(5)-\frac{8 \sqrt{3}}{243} \pi^{3} \zeta(3) .
$$

In particular when $l=0$ in (4.1), we can give some evaluation formulas for the double $L$-series attached to $\chi_{3}$. For example, putting $(k, l, d)=(2,0,2)$ in $(4.1)$ and using $\beta_{-1}(2,0 ; 1)=-\zeta(3)$ (see Example 2.3), we have

$$
\mathcal{L}\left(2,0,2 ; \chi_{0}, \chi_{3}\right)=-2 L\left(4, \chi_{3}\right)-\frac{\pi^{2}}{18} L\left(2, \chi_{3}\right)+\frac{26 \sqrt{3}}{81} \pi \zeta(3) .
$$

Using (3.14), we obtain (1.4).

\section{REFERENCES}

[1] T. Arakawa and M. Kaneko, 'On multiple $L$-values', J. Math. Soc. Japan (to appear).

[2] D.H. Bailey, J.M. Borwein and R. Girgensohn, 'Experimental evaluation of Euler sums', Experiment. Math. 3 (1994), 17-30.

[3] J.G. Huard, K.S. Williams and Z. Nan-Yue, 'On Tornheim's double series', Acta Arith. 75 (1996), 105-117.

[4] K. Matsumoto, 'On Mordell-Tornheim and other multiple zeta-functions', in Proceedings of the Session in analytic number theory and Diophantine equations (Bonn, January-June 2002), (D.R. Heath-Brown and B.Z. Moroz, Editors), Bonner Mathematische Schriften 360 (Mathematisches Institut der Universität Bonn, Bonn, 2003), pp. 17.

[5] L.J. Mordell, 'On the evaluation of some multiple series', J. London Math. Soc. 33 (1958), 368-371.

[6] D. Terhune, Evaluations of multiple L-values, (Ph.D. Thesis) (UT-Austin, 2002). 
[7] D. Terhune, 'Evaluations of double $L$-values', J. Number Theory 105 (2004), 275-301.

[8] L. Tornheim, 'Harmonic double series', Amer. J. Math. 72 (1950), 303-314.

[9] H. Tsumura, 'Evaluation formulas for Tornheim's type of alternating double series', Math. Comp. 73 (2004), 251-258.

[10] L.C. Washington, Introduction to the cyclotomic fields, (2nd ed.) (Springer-Verlag, New York, Berlin, Heidelberg, 1997).

Department of Management Informatics

Tokyo Metropolitan College

Akishima

Tokyo $196-8540$

Japan

e-mail: tsumura@tmca.ac.jp 